ing genetic effects where pooling of genes will occur, but it is not acceptable in assessing radiation carcinoma and leukaemia risk.

However, perhaps the worst anomaly lies in the collection of radiation records. Radiation workers in establishments subject to the Factories $\mathrm{Act}^{12}$ must, by law, have correct and accurate radiation records which are transferred with them when they change jobs. Other establishments such as universities and hospitals generally adopt the same procedure for their radiation workers, ${ }^{34}$ and recently the National Radiological Protection Board has set up a computerized record system which represents the first attempt to institute some form of central register.

The irony of the situation is that a large proportion of radiation workers will receive doses of less than 0.25 rem per year, this being the limit of measurement, and such doses will be carefully recorded. Doses obtained from medical and dental exposures for particular individuals on the other hand, which will in many cases considerably exceed these figures, will in general not be precisely known and will not be recorded. Such a situation is palpably absurd and it is obviously time that all radiation doses received by any individual from any source, whether it be occupational or from medical or dental diagnosis, should be accurately known and centrally recorded. Only when this is done can any reasonable evaluation of radiation risk be made.-I am, etc.,

\section{ROGER OLDHAM}

Department of Cardiology,

Groby Road Hospital, Leicester

1 Statutory Instruments, Factories, The Ionising Radiations (Sealed Sources) Regulations, 1969 SI No. 808). London, H.M.S.Q., 1969.

2 Statutory Instruments, Factories, The Ionising Radiations (Unsealed Radioactive Substances) Regulations, 1968 (SI No. 780). London, H.M.S.O., 1968

3 Department of Employment and Productivity Code of Practice for the Protection of Persons exposed to Ionising Radiations in Rese
Teaching. London, H.M.S.O., 1968.

- Department of Health and Social Security Code of Practice for the Protection of Persons against
Ionising Radiations arising from Medical and

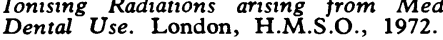

\section{Polyunsaturated Fatty Acids and Cancer}

SIR,-In your editorial "Are PUFA Harmful?" (6 October, p. 1) you mention the results of Pearce and Dayton, ${ }^{1}$ revealing an increased incidence of cancer in people taking a prudent diet enriched in polyunsaturated fatty acids (PUFA). Our previously reported results ${ }^{2-4}$ indicate a dose-dependent regulatory effect of PUFA on the antigeninduced lymphocyte response as measured with the macrophage electrophoretic mobility test (see fig.) and in the lymphocyte

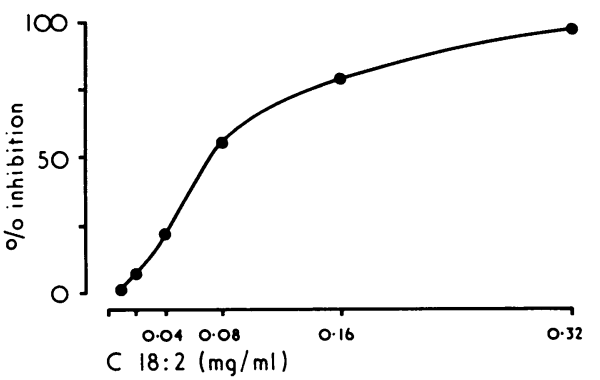

The inhibitory effect of various concentrations of linoleic acid (c 18:2) on the PPD-induced lymphocyte response, measured by the macrophage electrophoretic mobility test. transformation test. Therefore an increased serum PUFA level might suppress the reaction of the immune system to cancer and favour tumour development and growth. If further investigations confirm our findings then a therapeutic lowering of the serum PUFA level might be a supporting factor in the treatment of cancer. Owing to an inverse relationship in the metabolism of single unsaturated fatty acids and PUFA, as shown for example by Belin et al..$^{5}$ for oleic (C 18:1) and linoleic acid (C 18:2), such a decrease in the serum PUFA level can readily be produced by an increased C 18:1 intake.

In addition, there is now increasing evidence for an involvement of the immune system in the process of ageing and an immunoregulatory effect of PUFA may also be considered in premature ageing caused by excessive PUFA consumption. ${ }^{6}$ - I am, etc.

Medical Research Council,

J. MERTIN

Newcastle General Hospital,
Newcastle upon Tyne

Pearce, M. L., and Dayton, S., Lancet, 1971, 1, 464.

British, J., Shenton, B. K., and Field, E. J., British Medical fournal, 1973, 2, 777.

Mertin, J., Shenton, B. K., and Field, E. J. fournal of Neurological Sciences. In press.

Mertin, J., Hughes, D., Shenton, B. K., and
Dickinson, J. P., Klinische Wochenschrift. In press.

Belin. J., Pettet, N. Smith, A. D., Thompson, R. H. S., and Zilkha, K. J. Fournal of Neurology, Neurosurgery and Psychiatry, 1971, 34, 85, 723 .

\section{Anaesthetic Safety Devices}

SIR,-We have been following this correspondence with interest because we have been and still are actively working on the problem. Though our results are not yet ready for publication, our interim conclusions may be useful to others.

Dr. T. H. Howells's proposal (13 October, p. 105) for the use of the reserve cylinder is not new, and careful consideration has shown that it does not eliminate human error and could well make things worse. As Dr. Howells himself indicates, it is possible for both oxygen cylinders to become exhausted; the anaesthetist may fail to notice that he has transferred to the reserve cylinder, or may decide that it is not worth changing the cylinder till the end of the operation and then forget, or be prevented from doing so. He will then run out of gas and find he has no reserve. In addition, the adoption of Dr. Howells's system would require anaesthetists to make a substantial change in their habits, and the resulting confusion might well cause more accidents than it would prevent.

Dr. R. Parfitt (22 September, p. 635) and Dr. R. L. Wynne (13 October, p. 105) have both shown how unreliable oxygen failure alarms can be. Though a simpler and more reliable oxygen failure alarm might perhaps be made, it would not eliminate human error, because experience with all kinds of monitoring systems has shown repeatedly that audible alarms are liable to be switched off or otherwise put out of action when, as is bound to happen, they give a false alarm. In our opinion, therefore, there is only one approach to the problem which is likely to be effective and that is not to try to prevent oxygen failure, but to prevent asphyxia resulting from it.
Both Dr. Parfitt and Rosen and Hillard ${ }^{1}$ have included this requirement in their criteria for a "perfect" safety system, and as they have recently reported (20 October, p. 167) the latter's device is now being marketed, in a modified form, by the British Oxygen Company as a successor to the Bosun. We feel that some of their requirements are unnecessarily stringent and their device correspondingly unduly complex, so we have designed a simpler one which we believe is capable of preventing serious anoxia being produced by oxygen failure in almost all circumstances, and which can be fitted easily to any gas-and-oxygen anaesthetic machine. The device is a valve fitted between the patient and the machine which shuts him off from the machine and vents him to air if the oxygen pressure falls significantly. It also diverts the other anaesthetic gases through a separate vent, and both vents can be fitted with whistles. Thus if the patient is breathing, he will come to no harm and his whistle will draw attention to the oxygen failure. If he is on a ventilator, the other whistle will give warning long before serious anoxia occurs. A patient being ventilated with oxygen and air will also be safe, and only a paralysed patient being ventilated with pure oxygen will still be at risk. A device of this kind has been made and is now being evaluated, and we hope to be able to publish a full description and account of its performance shortly.-We are, etc.

H. T. DAvenPort B. M. WRIGHT

Northwick Park Hospital and Clinical Research Centre, Harrow, Middx

1 Rosen, M., and Hillard. E. K., British fournal of Anaesthesia, 1971, 43, 103.

\section{Selection of Patients for Clinical Trials}

SIR,-In advancing his thesis that the results of clinical trials may be influenced by the natural prognosis of the patients selected Dr. J. P. Leff (20 October, p. 156) refers to the recently reported M.R.C. multicentre trial of continuation thymoleptic medication in depressed patients. ${ }^{12}$ He suggests that the difference in outcome between Glasgow and the o her centres may be due to the inclusion of more "good prognosis" or less severely depressed patients in the Glasgow sample, taking as his index the differences in relapse rate in patients receiving placebo. He may indeed be correct, but I think there are other explanations for the differences between centres which deserve consideration.

Firstly, in the trial as a whole, relapse rates were much higher in those patients who were not completely well when maintenance therapy was commenced, especially in those patients who drew placebo. Fewer Glasgow patients had such residual symptoms and it is therefore not surprising that their relapse rates were lower. These figures may of course be open to other interpretations but it may well be that many patients were admitted to "maintenance" therapy too soon and were, in consequence, more likely to relapse.

Secondly, high relapse rates may also have been due to the failure of patients to take their medication, and this would have been so for those taking placebo as well as those on active drug. Tablet counts were 